\title{
Radionuclide Imaging of Infection: What the Future Holds
}

\author{
Christopher J. Palestro* \\ Albert Einstein College of Medicine of Yeshiva University and Division of Nuclear Medicine and Molecular \\ Imaging; North Shore Long Island Jewish Health System; Manhasset and New Hyde Park; NY USA; \\ palestro@lij.edu
}

\begin{abstract}
Nuclear Medicine plays an important role in the evaluation of patients suspected of harboring infection. Gallium imaging is especially useful for opportunistic infections and spinal osteomyelitis. In vitro labeled leukocyte imaging is the current radionuclide gold standard for imaging most infections, in immunocompetent patients, including cardiovascular, postoperative, and musculoskeletal infections (except spinal osteomyelitis). Several in-vivo leukocyte labeling methods have been investigated, but none are widely used. Results obtained with radiolabeled antibiotics have been disappointing. Data on FDG are still emerging, but this agent appears to be especially valuable in fever of unknown origin, spinal osteomyelitis, vasculitis and sarcoidosis. It is conceivable that in the near future, FDG-PET and PET/CT will replace gallium for many indications. Investigators also are studying ways to label leukocytes with positron emitters in order to combine the advantages of PET with those of labeled leukocytes.
\end{abstract}

Key words: Infection, labeled leukocytes, gallium, FDG, PET

\section{INTRODUCTION}

\section{Tracers}

Gallium-67

Several factors affect gallium uptake in inflammation. Inflammation is characterized by increased blood flow and increased vascular membrane permeability which result in increased delivery and accumulation of the plasma proteins to which gallium is bound. Bacteria are responsible for some gallium uptake in infection, both through direct tracer uptake and through siderophores. These low molecular weight metal chelates, produced by bacteria for iron binding and transport, are gallium-avid. Presumably, the siderophore-gallium complex is transported into the cell from which it cannot be released without destruction of the entire molecule. Plasma proteins also account for gallium uptake in inflammatory foci. Transported in the plasma bound to transferrin, gallium dissociates from transferrin at the site of inflammation and combines with lactoferrin. A small amount of gallium is also transported bound to leukocytes (Palestro, 1994).

\section{Labeled leukocyte}

Labeled leukocyte imaging is the radionuclide "gold standard" for imaging focal infection in the immunocompetent population. Leukocytes can be labeled with indium-111-oxine or with technetium-99m-exametazime. Uptake depends on intact chemotaxis, number of cells labeled, types of cells labeled, and the nature of the inflammatory response in a particular entity. The in- vitro labeling of leukocytes is a routine procedure and

\footnotetext{
${ }^{*}$ Author for correspondence
} 
does not affect the migratory response of the labeled cells. A minimum peripheral leukocyte count of $2000 / \mathrm{mL}$ is necessary for a satisfactory study. In most clinical settings the predominant cell type labeled is the neutrophil. The scan is, therefore, most sensitive for detecting neutrophil mediated inflammatory processes, i.e., bacterial infections. Labeled leukocyte imaging is less useful in illnesses that incite other than a neutrophilic response, i.e., sarcoid (Love et al., 2004).

\section{In-vivo leukocyte labeling agents}

Considerable effort has been devoted to developing in-vivo methods of labeling leukocytes, including peptides and antigranulocyte antibodies/antibody fragments. One agent is a murine monoclonal G1 immunoglobulin, BW 250/183, which binds to Nonspecific CrossReactive Antigen -95 present on neutrophils. Studies generally become positive by 6 hours after injection; imaging at 24 hours may increase lesion detection (Love et al., 2003)

Fanolesomab is a monoclonal murine $M$ class immunoglobulin that binds to $\mathrm{CD} 15$ receptors present on leukocytes. This agent presumably binds both to circulating neutrophils that eventually migrate to the focus of infection, and to neutrophils, or neutrophil debris containing CD15 receptors, already sequestered in the area of infection (Love et al., 2003).

Antibody fragments are appealing because, unlike the whole antibody, they do not induce a HAMA response. Sulesomab is a murine monoclonal antibody fragment of the $\mathrm{IgG} 1$ class that binds to Normal Cross-Reactive Antigen-90 present on leukocytes. The mechanisms of uptake include binding to circulating neutrophils and crossing permeable capillary membranes with binding of the fragment to leukocytes already present at the site of infection (Love et al., 2003).

\section{FDG}

Fluorine-18- fluorodeoxyglucose (FDG) is transported into cells via glucose transporters, and is phosphorylated to ${ }^{18} \mathrm{~F}-2$ '-FDG-6 phosphate but is not metabolized. The degree of cellular uptake of FDG is related to the cellular metabolic rate and to the number of glucose transporters. Activated inflammatory cells, such as neutrophils, lymphocytes, monocytes, and macrophages, demonstrate increased expression of glucose transporters and in inflammatory conditions, the affinity of glucose transporters for deoxyglucose presumably is increased by various cytokines and growth factors (Love et al., 2005). Investigators now are studying ways to label leukocytes with positron emitters to combine the advantages of PET with those of labeled leukocytes (Bhargava et al., 2008, Rini et al., 2006).

\section{Radiolabeled Antibiotics}

Radiolabeled antibiotics have none of the disadvantages of the in-vitro labeled white cell or antigranulocyte antibody procedures. Presumably, the radiolabeled antibiotic is incorporated and metabolized by bacteria present in the infectious focus resulting in accurate and specific localization of the infection. The most extensively studied of these compounds is technetium-99mciprofloxacin Published results have been variable. (Palestro et al., 2008).

\section{Indications}

\section{Opportunistic Infections}

The opportunistic infection that most often affects the brain of the AIDS patient is toxoplasmosis. Anatomic modalities may demonstrate focal brain lesions, but it is not always possible to differentiate CNS lymphoma from toxoplasmosis in these patients. FDG-PET is useful for this purpose. Hypermetabolic lesions on FDG-PET images are indicative of lymphoma, while hypometabolic lesions are indicative of toxoplasmosis (Love et al., 2005).

Gallium imaging is the radionuclide procedure of choice for AIDS-related respiratory tract diseases. A normal gallium scan of the chest excludes infection with a high degree of certainty, especially in the setting of a negative chest radiograph. In patients with known Kaposi's sarcoma, however, a negative gallium scan and an abnormal chest $\mathrm{x}$-ray are strong evidence that the patient's respiratory disease is tumor related. Lymph node uptake of gallium in the HIV (+) patient is most often due to mycobacterial disease or lymphoma. Focal, or localized, pulmonary parenchymal uptake of gallium is usually associated with bacterial pneumonia. Diffuse pulmonary gallium uptake may be due to numerous causes although the more intense the uptake, the more likely the patient is to have Pneumocystis carinii pneumonia (Palestro, 1994). 
Labeled leukocyte scintigraphy has a limited role in the work-up of AIDS-related thoracic diseases because it is not sensitive for detecting opportunistic infections. Labeled leukocyte imaging, however, is very useful in the detection of AIDS-related colonic infections (Palestro et al., 1995).

\section{Fever of undetermined origin}

Fever of undetermined origin (FUO) is an illness of at least three weeks duration, with several episodes of fever exceeding $38.3{ }^{\circ} \mathrm{C}$, and no diagnosis after an appropriate inpatient or outpatient evaluation. Identifying the cause of an FUO is not an easy task, and no single test provides the diagnosis in all patients. Nevertheless radionuclide studies provide important information. Traditionally, gallium and/or labeled leukocyte imaging have been used. FDG-PET is an intriguing and exciting alternative to the conventional radionuclide approach to the FUO. FDG is similar to gallium; i.e., though not specific, it is exquisitely sensitive, ideally suited to the evaluation of an entity with diverse etiologies. The short half-life of ${ }^{18} \mathrm{~F}$, moreover, does not delay the performance of any additional radionuclide studies that might be contemplated. Recent investigations have found that the test is sensitive, compares favorably to, and could potentially replace Ga-67 for the evaluation of patients with FUO. The value of FDG-PET is further enhanced by data, which suggest that vasculitis and bacterial endocarditis, both of which can be the source of an FUO, and which are not amenable to detection with other radionuclide studies, may be identified with this test. Other entities including thromboembolic disease, sarcoidosis, and chronic granulomatous disease, which can all present as an FUO, are also associated with increased FDG uptake (Love et al., 2005).

The negative predictive value of FDG-PET in the patient with an FUO is apparently very high; i.e., a negative test makes it very unlikely that a morphological origin of the fever will be identified. If confirmed in future investigations, FDG-PET, by reducing the number of imaging studies performed, may prove to be a very cost effective method of investigating the FUO (Love et al., 2005).

\section{Postoperative infection}

Anatomic modalities such as ultrasonography and computed tomography cannot always distinguish an abscess form other fluid collections, and on occasion, even from normal postoperative changes. The surgically distorted anatomy and metallic sutures can further complicate the interpretation of these studies. Radionuclide tests are an adjunct to anatomic imaging modalities and facilitate the differentiation of abscess from other fluid collections, from tumor, and even from normal postoperative changes. Although gallium can detect intra-abdominal infection, the normal presence of large bowel activity can obscure foci of infection; the need to wait 48 hours or more between injection and imaging is another disadvantage. For these reasons ${ }^{111}$ In -labeled leukocyte scintigraphy is the preferred radionuclide study. The overall accuracy of labeled leukocyte imaging in the febrile postoperative patient is about $85-90 \%$. There are few data available about the role of FDG-PET for this indication; uptake of this tracer in normally healing organs and surgical incisions raises questions about its role in the postoperative patient (Palestro et al., 2000; Love et al., 2005).

\section{Cardiovascular infection}

Echocardiography accurately diagnoses bacterial endocarditis, and radionuclide methods play a very limited role in the diagnostic workup of this entity Echocardiography is less sensitive, however, for detecting myocardial abscesses. Both gallium and labeled leukocyte imaging have successfully detected myocardial abscesses in patients with infective endocarditis. In one series labeled leukocyte imaging correctly identified three myocardial abscesses, none of which were detected with echocardiography.

A serious complication of bacterial endocarditis is the mycotic aneurysm. The patient with a suspected mycotic aneurysm is best managed by combining anatomic imaging, to localize the aneurysm, with labeled leukocyte imaging, to determine whether or not infection is present (Palestro et al., 1999).

Labeled leukocyte imaging is the radionuclide procedure of choice for diagnosing prosthetic vascular graft infection. The sensitivity exceeds $90 \%$ and neither duration of symptoms nor pretreatment with antibiotics adversely affect the study. The specificity is more variable ranging from $53 \%$ to $100 \%$. False positive results are associated with perigraft hematomas, graft thrombosis, bleeding, and pseudo-aneurysms (Palestro et al., 1999). 


\section{Central nervous system}

Labeled leukocyte scintigraphy reliably differentiates cerebral abscess from tumor. Faint or moderate uptake in tumors is occasionally observed. High dose steroid treatment can decrease study sensitivity (Palestro et al., 1999).

\section{Musculoskeletal Infection}

Except in the spine, labeled leukocyte imaging is the procedure of choice for diagnosing complicating osteomyelitis. To maximize accuracy, the test is frequently performed together with ${ }^{99 \mathrm{~m}} \mathrm{Tc}$ sulfur colloid marrow imaging. Labeled leukocytes accumulate in the bone marrow. Unfortunately, the "normal" distribution of hematopoietically active bone marrow is variable. Systemic diseases such as sickle cell and Gaucher disease produce generalized alterations in marrow distribution, while fractures, orthopedic hardware, and the neuropathic joint cause localized alterations. The normal distribution of hematopoietically active marrow in children varies with age. Consequently, it may not be possible to determine if an area of activity on a labeled leukocyte image represents infection or marrow. Performing complementary bone marrow imaging with ${ }^{99 \mathrm{~m}} \mathrm{Tc}$ sulfur colloid overcomes this problem. Both labeled leukocytes and sulfur colloid accumulate in the bone marrow; leukocytes also accumulate in infection; sulfur colloid, however, does not. The combined study is positive for infection when activity is present on the labeled leukocyte image without corresponding activity on the sulfur colloid marrow image. Any other pattern is negative for infection. The overall accuracy of combined leukocyte/marrow imaging is approximately 90\% (Love et al., 2001; Palestro et al., 2002; Palestro et al., 2007).

Labeled leukocyte imaging is not useful for detecting spinal osteomyelitis because $50 \%$ or more of these cases present as areas of decreased or absent activity. This photopenia is not specific for vertebral osteomyelitis, and is associated with other entities such as tumor, infarction, and Paget's disease (Palestro et al., 2007).

\section{Inflammatory bowel disease}

Technetium labeled leukocytes is the radionuclide agent of choice for inflammatory bowel disease (IBD), a group of disorders that include Crohn Disease and ulcerative colitis. Leukocyte imaging is very sensitive for detecting IBD, and can be used a screening test. The test is also useful in patients who refuse endoscopy or contrast radiography, and in those in whom these studies cannot be satisfactorily performed because of narrowing of the bowel lumen. The ability of the radionuclide study to differentiate active inflammation from scarring can have a significant impact on patient management. Labeled leukocyte imaging can also be used to monitor response to therapy. Imaging at multiple time points and SPECT increase the sensitivity of the test. Physiologic bowel activity, probably due to hepatobiliary excretion of $99 \mathrm{~m}$ Tc-labeled hydrophilic complexes, frequently appears on delayed images, and must be differentiated from activity secondary to inflammation (Palestro et al., 2006).

There are limitations to labeled leukocyte imaging. It cannot define anatomic changes such as strictures, which are best delineated with endoscopy and contrast radiography. The test is less sensitive for upper, than for lower, gastrointestinal tract disease. The sensitivity of the test also may be affected adversely by concomitant administration of corticosteroids (Palestro et al., 2006).

\section{CONCLUSION}

In vitro labeled leukocyte imaging, for the foreseeable future, will remain the radionuclide gold standard for imaging most infections in immunocompetent patients. Investigators are studying ways to label leukocytes with positron emitters to combine the advantages of PET with those of labeled leukocytes. FDG-PET and PET/CT will probably replace gallium for imaging infection and inflammation.

\section{RESUMO}

A Medicina Nuclear tem um importante papel na avaliação de pacientes suspeitos de apresentar infecção. A imagem obtida com gálio é especialmente útil para infecções oportunistas e osteomielite espinal. Imagem de leucócitos marcados in vitro com radionuclídeo é o padrão ouro atual para imagem de muitas infecções, em pacientes imunocompetentes, incluindo os cardiovasculares, pós-cirúrgicos, e com infecções músculo-esqueléticas (exceto osteomielite 
espinal). Vários métodos de marcação de leucócitos in vivo têm sido investigados, mas nenhum é amplamente utilizado. Resultados obtidos com antibióticos radiomarcados têm sido decepcionantes. Os dados sobre FDG são ainda emergentes, mas esse agente parece ser especialmente valioso em febre de origem desconhecida, osteomielite espinal, vasculite e sarcoidose. É concebível que em futuro próximo, FDG-PET e PET/CT irão substituir o gálio para imagem de infecção e inflamação. Investigadores estão estudando modos de marcar leucócitos com emissores de pósitron para combinar as vantagens do PET com aquelas dos leucócitos marcados.

Palavras chaves: Infecção, Leucócitos marcados, Gálio, FDG, PET.

\section{REFERENCES}

Bhargava, K. K.; Gupta, R. K.; Love, C. (2008), Human leukocyte labeling with ${ }^{64} \mathrm{Cu}$ : An intraindividual comparison with ${ }^{111}$ In-oxine and ${ }^{18}$ F-FDG. $\quad J ~ N u c l$ Med., 49, 133.

Love, C.; Tomas, M. B.; Marwin, S. E.; Pugliese, P. V.; Palestro, C. J. (2001), Role of nuclear medicine in diagnosis of the infected joint replacement. RadioGraphics, 21, 1229-1238.

Love, C.; Palestro, C. J. (2003), 99mTc-fanolesomab. IDrugs., 6, 1079-1085.

Love, C.; Palestro, C. J. (2004), Radionuclide imaging of infection. J Nucl Med Tech., 32, 47-57.

Love, C.; Tomas, M. B.; Tronco, G. G., Palestro, C. J. (2005), FDG PET of infection and inflammation. RadioGraphics, 25, 1357-1368.
Palestro, C. J. (1994), The current role of gallium imaging in infection. Sem Nucl Med., 24, 128-141.

Palestro, C. J.; Goldsmith, S. J. (1995), The use of gallium and labeled leukocyte scintigraphy in the AIDS patient. Q J Nucl Med., 39, 221-230.

Palestro, C. J.; Torres, M. A. (1999), Radionuclide imaging of nonosseous infection. Q J Nucl Med., 43, 46-60.

Palestro, C. J.; Love, C.; Tronco, G. G.; Thomas, M. B. (2000), Role of radionuclide imaging in the diagnosis of postoperative infection. RadioGraphics, 20, 16491660.

Palestro, C. J. (2002), Radionuclide imaging of the painful joint replacement: past, present, and future. Braz Arch Biol Tech., 45, 9-14.

Palestro, C. J.; Love, C. (2006), Scintigraphic imaging of inflammation and inflammation. In: Fundamentals of Diagnostic Radiology, $3^{\text {rd }}$ ed. Brant, W. E.; Helms, C. A. Lippincott Williams and Wilkins, Philadelphia, pp. 1436-1451.

Palestro, C. J.; Love, C. (2007), Radionuclide imaging of musculoskeletal infection. Braz Arch Biol Tech. 50, 15-27.

Palestro, C. J.; Love, C. (2008), Nuclear Medicine and diabetic foot infections. Sem Nucl Med., in press.

Rini, J. N.; Bhargava, K. K.; Tronco, G. G.; Singer, C.; Caprioli, R.; Marwin, S. E.; Richardson, H. L.; Nichols, K. J.; Pugliese, P. V.; Palestro, C. J. (2006), ${ }^{18}$ F-FDG in vitro labeled leukocytes versus ${ }^{111} \mathrm{In}$ labeled leukocytes for detection of infection. Radiology, 238, 978-987.
Received: August 18, 2008; Accepted: September 01, 2008. 